\title{
The promotion of Teaching Chinese Medicine in Higher Vocational College in Implicit Moral Education
}

\author{
Lv Guifeng \\ Traditional Chinese Medicine Department \\ Langfang Health Vocational College \\ Lang Fang, China \\ e-mail: lgflf@sohu.com
}

\author{
Wang Juan \\ English Department \\ Langfang Health Vocational College \\ Lang Fang, China \\ e-mail: wangjuan407@sohu.com
}

\begin{abstract}
According to the features of Higher Vocational College (HVC) and the facts of the students, Implicit Moral Education (IME) became an important way to strength the actual effect of the Moral Education (ME). Classroom teaching is one of efficient forms to infiltrate IME. Chinese Medicine (CM) can be traced to Chinese ancient civilization that contains plain philosophical ideas. The humanities expressed by the teaching process influenced subtly on promoting IME. The influences are the followings: to inspire students' patriotism and enhance a sense of national pride; to develop the spirit of teamwork and the consciousness to adjust to society with positive attitude; to achieve healthy doctors' ethic and the humanitarianism of life-saving; improving the personal qualities..
\end{abstract}

Keywords- Teaching Chinese Medicine (TCM); Implicit Moral Education (IME); Higher Vocational College Students (HVCS)

"Some suggestions on improving the teaching quality of HVC of the PRC Ministry of Education” clearly says HVC should insist on educated-oriented and ME for first. The basic task is to develop skilled and moral students....HVC is a special college which not only develop the applied staff with good professional knowledge and skills but also strength ME under the situation. Consider it as a great task to develop high-quality students with healthy professional ethic. Although explicit moral education is in the first place in a variety of ME ways, to make a good use of IME is still an effective way ${ }^{[1]}$. There are many forms in IME, but teaching process with the mutual action play an important role in IME. "China Educational Reform and Development Outline" pointed out that ME should be infiltrated into and be throughout the whole teaching process. $\mathrm{CM}$ is a required course of some majors in health HVC. The long history, rich contents and deep philosophical ideas showed in TCM will be infiltrated into the IME naturally.

\section{THE ME INFILTRATION IN CLASSROOM TEACHING IS AN IMPORTANT WAY TO PUT IME INTO PRACTICE EFFICIENTLY IN HVC}

Higher Vocational Education is a special higher education, whose student resources are various. Some are graduated from senior school; some are graduated from junior school; some are graduated from secondary vocational school. The quality of these students is greatly different, so their ME is a hard task. These students who are grown up under rich materials were born in the early 90's. However they are influenced by some problems such as the social competition and pressure, the safe problems of society, food, communications and internet. They are having distinctive personality, strong independent ideas and rebellious and critical thoughts. Each year the ideological education is added to the teaching plan in HVC and some ideological education activities are organized. But these education cannot work actually moreover make students psychological inversion. To some extend, therefore, IME as effective way is more suit to the students' mental need.

Implicit morality is activities performed by educator indirectly and impliedly. It refers to an educational way by which the activities, the cultural programs, the rules and regulations and interpersonal relationships in campus not including normal teaching plan influenced the students' view of world and life, their value and their thoughts. The classroom is the main place of teacher-student communication. The mutual interaction showed by teacher's teaching and students' learning in teaching process. So a positive relationship of harmonious and trust is formed. The major teaching is the main task for HVC. The students are active to learn major course. So students are willing to learn from their major teachers. When the teacher can show information of ME naturally in his her teaching, students, the subjectivity of learning who can accept and participate spontaneously are educated naturally. The major teachers can teach major knowledge and skills, in addition, their personal qualities and behaviors can greatly influence on students' thoughts. Therefore teachers in HVC should improve personal qualities unceasingly and become a model of students and have an active influence on students. This influenced unconsciously IME can promote the effect of ME in HVC.

\section{FULLY PLAY CM’S HUMANITIES FEATURES TO PROMOTE IME}

$\mathrm{CM}$ has a long development history, which is gathering precious experience by fighting with diseases in a long term. It made a great contribution to national health. It is also important components of the good Chinese traditional culture. The deep and long-standing medical ethic lead the current medical students to build correct the views of life and values. TCM is a process both teaching medical knowledge and skills and an internal process of ME. 


\section{A. To inspire students' patriotism to strengthen national pride}

Our ancestors formed original medical knowledge which combined treating, curing, caring and keeping. CM which developed unceasing under the generations' hard work constructed unique medical theory and diagnostic system. The great contributions for human health made by CM are known all over the world. "Huang Di Nei Jing" from Warring States to Qin-Han recorded that the length rate between esophagus and intestine was 1:35 (Modern Anatomy 1"37). "Wei Rong Na Shui Gu San Dou Wu Sheng" indicates the digestive function of intestine tract played an important role in body's work. "Xin Zhu Shen zhi Xue Mai" "2] told that blood flows round the body in vessel endlessly. (It was more 1000 years earlier than the blood circulation discovered by Harvey from England in 1628). It proved that at that time Chinese Anatomy knowledge was in a leading position in the world. "Ma Fei San" which was used in surgical operation made by Hua Tuo, a famous doctor in Han Dynasty was the earliest anesthetic in the world. "Xin Xiu Ben Cao" of Tang Dynasty was the first pharmacopoeia. "Ben Cao Gang Mu” of Ming Dynasty, which was translated into 17 languages, was called Oriental Great Pharmacopoeia and made great contributions to the development of world medicine. The smallpox inoculation used by doctors in Ming Dynasty was spread to Russia, Korea, Japan and Turkey and so on. (It was more 200years earlier than England), which became the pioneer in immunological world. The more than 2000-year development of $\mathrm{CM}$ is the essence of wisdom of Chinese people. The students can realize the ever leading position and great contributions of CM in the world by learning CM. Thus students are inspired patriotism and strengthened national pride. They will make their minds to study hard, carry forward and innovate for national health cause.

\section{B. To develop the teamwork to adjust to the society with positive attitude}

The concept of holism is one of CM's theory system features, which is CM's understanding in body's wholeness and unity among human, nature and social environment. According to CM, our body is a whole which is made of five physiological systems. They are heart system, liver system, spleen system, lungs system and kidney system. Any part of each system is a component of the whole. The supplementrestrict each others five systems keep a dynamic balance. Our body is in a physiological state. If one of them cannot work smoothly the others are influenced and cannot keep the balance. Our body is in pathological state. So only five systems are on duty and co-ordinate the body can keep healthy state. To the unity between human and social environment, the view of CM is that as individual the person belongs to the society. The person's social status, economic status, cultural background and interpersonal relationship can arouse various changes in physiology, psychology and pathology. Good social environment which make people lift spirit and innovate can help physical and mental health. Bad environment made people depressed and tension can harm health. People should avoid bad environment and adjust mood to improve the adaptability to society. So we can keep fit.

The current HVS (higher vocational students) are growing in the time when information is developing rapidly. They are experiencing the latest and lead to the future. Internet which is known in their junior or primary school is the closest partner in their daily lives. They can get rich learning materials, go shopping, talk with friends and strangers, and express their feelings and opinions on the internet. Their real world for living and communicating gets smaller under the unreal condition. They are the only child in their families. With the reducing relatives made them alone. They are indifference with the family relationship. Internet became necessary and reliable in their lives, which are changing students' ideas. They had different values and living ways themselves. ${ }^{[3-4]}$ They are fashionable, creative but sharp, eccentric, self-centered. Compared to common college students, they have a little knowledge, bad habits and are indifference with the collective. Therefore the education and guidance from school in this special period will play an important role in correcting students' values, views on world and life.

The plain philosophical ideas included in CM holism can make students understand that they are living in collective and their behaviors can influence collective interests. Wherever they go they belong to society. They cannot change the society. They only adjust themselves to it actively. Although some people are capable they cannot achieve what they want because they are in a rich division society. Maybe they succeed they paid a lot that we cannot stand. So teamwork is necessary to achieve in real life. Teamwork need cohesion and fighting force so that each one should cooperate well with others thought there are many difference in a team. We were excited when spaceship went up to sky. We cheered when players won golden medals. We knew the fact that these are fruits of teamwork. Living in society people should build up good social environment and gain strong support so that they can success.

\section{To shape health medical ethic and life-saving in humanitarianism}

$\mathrm{CM}$ was developed with Chinese civilization which is one of the oldest in the world. In ancient China, the moral standard is kind, justice, courtesy, wise and honest. It had a great influence on CM. The standard was abided by generations' doctors. Qin Yueren expressed his medical ethic by saving the prince. The Chinese idioms 'xinglinchunnuan' ‘yimanxinglin' praised Dong Feng, a famous doctor during Sanguo. Sun Simiao, famous doctor of Dang Dynasty explained medical ethic in "Da Yi Jing Cheng" which are respected and spread by generations' doctors. "Ben Cao Gang Mu' wrote by Li Shizhen in Ming Dynasty pointed out doctors should treat patient with kindness and love all. These medical ethic passed by generations' doctors tells us how to be a man as well as to be a doctor.

The current college students got much more information faster than ever with internet. They are moved by medical staffs that are saving people in dangerous spots. They are proud of the miracles worked by doctors who overcame lot 
difficulties. They knew evil trend of the internal medicine field. Patients were dead by careless doctors. Without gained profit patients cannot be taken good care of. Patients will paid more because of others' fees. These made bad expressions to students who are looking forward to society. Their ideas and beliefs formed just are influenced. Teachers should critical theses unhealthy bravely and correct students' values. TCM is a process that combined teaching and education. On one hand, students should understand that lofty medical ethic as Chinese traditional virtue is the base of excellent medical staff by presenting famous doctors in history. On the other hand, combining social facts and analyzing correctly actual problems such as hard to see a doctor, expensive to see a doctor and frequent doctor- patient issues, teacher made student know life-saving is bounded duty of medical staff under any social system or any social situation. Medical staff should be self-sacrifice, be responsible, be non-difficulty, be self-disciplined, be honest and hard-working for human health tirelessly. ${ }^{[5]}$

\section{To gather thoughts to improve personal qualities}

In CM our moods such as happiness, anger, anxiety, thinking, sorrow, fear and surprise are showed in daily life. As usual these moods cannot cause disease. But a strong and lasting mood which cannot be accepted by body will cause disease or disease recurrence. The change of mood has an influence on patients' condition. The positive is helpful to recover. The passive will further the disease. In addition, the change of social environment, for example, greatly change in social and economic status, family issues, the death of family member, tense relations among colleagues can break the balance kept in physiology and psychology. Under this condition there is something wrong in body and mood.

Great pressure on the students born in 90s, they who are taken to great hopes and good cared by parents are struggling under Chinese education system. Teachers paid more attention to teaching knowledge and little to EM before becoming a college student so that most of college students are poor psychological tolerance, depressed after failures, great difference in mood change, radical in dealing with issues, eccentric and unreasonable. In TCM, teachers should explain clearly the harm to health with bad moods combining each own matters while teachers should teach students to control their moods naturally. People should be generous, be self-controlled, know others well, keep calm. Containing education in teaching naturally can make students moral ideas changed. The changes in ideas will take place gradually with students' growth. It is hard to know oneself well in current develop-rapidly society, which became more impetuous by expanding desires. The deep-cultural plain philosophical ideas from CM formed into Chinese ancient civilization can clean your thoughts comfortable.

Notes

Ministry of education. "Some suggestions on improving the teaching quality of HVC" (Higher Education No.16, 2006). 2006-11-26

Xi Caiyun. "Extension IME to Reform of Higher Education School" [J]. 'Party Construction and Idea Education in school’, 2006(1): 27.

\section{ACKNOWLEDGMENT}

TCM influenced generations' medical learners with its profound cultural background. The teachers of CM who devoted themselves to medical studying should make students think and understand what learned under teacher' education and influence according to the features of HVS. Thus there will be promotion in optimizing ME of the colleges.

\section{REFERENCES}

[1] Feng Guang. Developing IME by Grasping Features: The Real Ways to improve Effectiveness of IME[J]. Education and Profession, 2009 (35): 19-21

[2] Sun Guangren. Chinese Medicine [M]. Beijing: China Chinese Medicine Publishing House, 2002: 3.

[3] Jiang Xueyan, Tian Huijing. The Features of the Generation after 90s and Their Values Education [J]. Theory Research, 2011 (5): 173-174.

[4] Zhang Tao, Wu Chengcheng, Kong Shuai. Understanding the Features of the Generation after 90s and strengthening Ideological Education Efficiency [J]. China Urban Economy, 2012 (1): 86.

[5] Zhang Bin. The Infiltration of Moral Education in Teaching Chinese Medicine in New Period [J]. China Education Innovation Herald, 2009 (36): 19 